\title{
A possible influence of the Great White Spot on Saturn kilometric radiation periodicity
}

\author{
G. Fischer ${ }^{1,2}$, S.-Y. Ye ${ }^{2}$, J. B. Groene ${ }^{2}$, A. P. Ingersoll ${ }^{3}$, K. M. Sayanagi ${ }^{4}$, J. D. Menietti ${ }^{2}$, W. S. Kurth ${ }^{2}$, and \\ D. A. Gurnett ${ }^{2}$ \\ ${ }^{1}$ Space Research Institute, Austrian Academy of Sciences, Schmiedlstr. 6, 8042 Graz, Austria \\ ${ }^{2}$ Department of Physics and Astronomy, The University of Iowa, 203 Van Allen Hall, Iowa City, Iowa 52242, USA \\ ${ }^{3}$ Division of Geological and Planetary Sciences, 150-21, California Institute of Technology, Pasadena, California 91125, USA \\ ${ }^{4}$ Department of Atmospheric and Planetary Sciences, Hampton University, 23 E. Tyler Street, Hampton, Virginia 23668, USA
}

Correspondence to: G. Fischer (georg.fischer@oeaw.ac.at)

Received: 20 August 2014 - Revised: 4 November 2014 - Accepted: 5 November 2014 - Published: 4 December 2014

\begin{abstract}
The periodicity of Saturn kilometric radiation (SKR) varies with time, and its two periods during the first 5 years of the Cassini mission have been attributed to SKR from the northern and southern hemisphere. After Saturn equinox in August 2009, there were long intervals of time (March 2010 to February 2011 and September 2011 to June 2012) with similar northern and southern SKR periods and locked SKR phases. However, from March to August 2011 the SKR periods were split up again, and the phases were unlocked. In this time interval, the southern SKR period slowed down by $\sim 0.5 \%$ on average, and there was a large jump back to a faster period in August 2011. The northern SKR period speeded up and coalesced again with the southern period in September 2011. We argue that this unusual behavior could be related to the so-called Great White Spot (GWS), a giant thunderstorm that raged in Saturn's atmosphere around that time. For several months in 2011, the visible head of the GWS had the same period of $\sim 10.69 \mathrm{~h}$ as the main southern SKR modulation signal. The GWS was most likely a source of intense gravity waves that may have caused a global change in Saturn's thermospheric winds via energy and momentum deposition. This would support the theory that Saturn's magnetospheric periodicities are driven by the upper atmosphere. Since the GWS with simultaneous SKR periodicity measurements have only been made once, it is difficult to prove a physical connection between these two phenomena, but we provide plausible mechanisms by which the GWS might modify the SKR periods.
\end{abstract}

Keywords. Magnetospheric physics (planetary magnetospheres) - meteorology and atmospheric dynamics (atmospheric electricity) - solar physics astrophysics and astronomy (radio emissions)

\section{Introduction}

Saturn kilometric radiation (SKR) was detected by the Voyager spacecraft as a strong auroral radio emission peaking in the frequency range of a few hundred $\mathrm{kHz}$. It is believed to be generated by unstable electron distributions in the auroral zones via the cyclotron maser instability. SKR shows two polarizations, it is mostly right-hand polarized when observed from the northern hemisphere, and left-hand polarized when observed from the southern hemisphere (Kaiser et al., 1984; Lamy et al., 2008a). During the Voyager 1 approach to Saturn, the SKR modulation period was determined by Desch and Kaiser (1981) to be $10 \mathrm{~h} 39 \mathrm{~min} 24 \pm 7 \mathrm{~s}$ (10.6567 h). The slightly modified period of $10 \mathrm{~h} 39 \mathrm{~min} 22.4 \mathrm{~s}(10.6562 \mathrm{~h})$ was later adopted as Saturn's internal rotation period linked to the planetary magnetic field (Davies et al., 1996). Observations by the Ulysses and the Cassini spacecraft (Galopeau and Lecacheux, 2000; Gurnett et al., 2005) have shown that the SKR period varies of the order of $\sim 1 \%$ over the years. Given Saturn's massive moment of inertia, such a change is far too large to arise from a change in the rotation rate of the planet's deep interior (Gurnett et al., 2007). From 2004 until early 2009, SKR had shown two periods of 10.8 and $10.6 \mathrm{~h}$ (Kurth et al., 2008), attributed to SKR radiated from 
the southern and northern hemisphere, respectively (Gurnett et al., 2009). The periods converged during 2009, but so far there is no consensus when the SKR periods crossed or coalesced. Gurnett et al. (2010) suggested that the periods coalesced in spring 2010, similar to Lamy (2011), who suggested a crossing of two very close periods around the same time in spring 2010. The question is related to the proper identification of northern and southern SKR, where the wave polarization and the spacecraft latitude can be used to separate northern from southern SKR intensities. Fischer et al. (2014) suggested that northern and southern periods crossed in late August 2009, just a few weeks after Saturn equinox on 11 August 2009. Nevertheless, all authors (Gurnett et al., 2010; Lamy, 2011; Fischer et al., 2014) agree that northern and southern periods are very similar $(\sim 10.7 \mathrm{~h})$ in spring 2010. In contrast to that, the periods of magnetic field oscillations of both hemispheres did not cross in the time interval studied until late 2012 (Provan et al., 2013).

Another tracer of Saturn's magnetospheric rotation is Saturn narrowband radio emission observed at a frequency around $5 \mathrm{kHz}$. They are thought to be ordinary mode emissions, which are mode converted from Z-mode waves at density gradients, and radio wave direction-finding results point to a source in the auroral region (Ye et al., 2010a). They exhibit a dual periodicity with two components equal to the SKR periods until mid-2010 (Ye et al., 2010b), and both periods can be observed in both hemispheres. It is thought that the radiation can propagate from one hemisphere to the other as Z-mode waves before being mode-converted to escaping $\mathrm{O}$-mode radiation.

It is not known what causes the time-varying double periodicity of SKR and Saturn narrowband radio emissions. Similarly, time-varying double periodicities have been found for magnetic field oscillations (Andrews et al., 2011, 2012) and energetic electron fluxes (Carbary et al., 2009, 2011). Recent models suggest a rotating magnetic cam field structure (Southwood and Kivelson, 2009) or a centrifugally driven convective instability in the equatorial plasma disc of Saturn's inner magnetosphere (Gurnett et al., 2007) to be responsible for the temporal variation. A common feature of these models are currents aligned with Saturn's magnetic field that close in the plasma disc and Saturn's ionosphere, thereby linking the magnetosphere to the conductive part of Saturn's upper atmosphere. The rotation of the magnetosphere is maintained by the torque exerted by neutral-ion collisions in the upper atmosphere, and the torque is transmitted to the magnetosphere by these field-aligned currents (Huang and Hill, 1989). The currents could be influenced by seasonal changes in wind speeds or Pedersen conductivities in the ionosphere (Gurnett et al., 2009). A changing neutral wind in the auroral ionosphere should therefore have an influence on the slippage of the magnetosphere. A recent model has imposed double vortices in Saturn's auroral ionosphere on one or both hemispheres $(\sim 1000 \mathrm{~km}$ above the $1 \mathrm{mbar}$ level) that drive the field-aligned currents. It successfully reproduced many magnetospheric features (Jia et al., 2012; Jia and Kivelson, 2012). Another model used an asymmetric heating source in Saturn's thermosphere to drive winds and magnetospheric currents (Smith, 2011), but it could not reproduce the observed magnetic field perturbations.

In this paper, we will give some support to the hypothesis of an atmospheric origin of Saturn's magnetospheric periodicities by showing a temporal coincidence of specific SKR modulation features with the occurrence of the so-called Great White Spot (GWS). A GWS is a planetary-scale disturbance in Saturn's atmosphere (Sánchez-Lavega et al., 2011, 2012) that usually happens once per Saturn year (29.5 Earth years). It is a big thunderstorm that produces radio emissions caused by lightning discharges (Fischer et al., 2011). We will discuss the physical origin of the possible relation between the GWS and the SKR in Sects. 4 and 5. Before that, we will show the modulation of the common SKR signal and of Saturn narrowband radio emissions in Sect. 2, and we will also separate the SKR by polarization to derive the periods of the northern and southern SKR. In Sect. 3 we will take a detailed look at the SKR behavior during the GWS by plotting the northern and southern SKR phases with time and comparing them to the start, evolution, and demise of the spectacular GWS event.

\section{SKR and narrowband radio emission modulation spectrograms}

The normalized peak-to-peak power of the SKR and narrowband emission modulation is calculated with a method called tracking filter analysis, which was thoroughly described by Gurnett et al. (2009, 2011). The analysis uses the integrated intensity of the radio waves (integration over $10 \mathrm{~min}$ in time and over $80-500 \mathrm{kHz}$ in frequency for SKR, and 3-8 kHz for the narrowband emissions) as measured by the Cassini Radio and Plasma Wave Science (RPWS) instrument (Gurnett et al., 2004). The integrated intensity is distance-normalized by dividing by the average intensity over one Saturn rotation. The resulting normalized intensities are multiplied by a Hanning weighting function with a duration of 240 days. For the spectrum analysis, the normalized SKR intensities are sorted and averaged in $1^{\circ}$ longitude bins for a series of assumed rotation rates ranging from 785 to $830^{\circ} \mathrm{day}^{-1}$. The resulting averages are fit to a sinusoidal function of longitude whose squared peak-to-peak amplitude is proportional to the so-called normalized peak-to-peak power represented in the modulation spectrograms. The 240-day Hanning window is shifted in 30-day steps with a spectrum analysis for each step leading to a temporal resolution of about 12 measured periods per year. This periodicity analysis is applied to the complete SKR signal (Fig. 1), to the narrowband radio emissions (Fig. 2), and to the SKR signals of each hemisphere separated by wave polarization (Fig. 3). Northern SKR was defined as strong SKR $(\mathrm{SNR}>10)$ with circular polarization $d_{\mathrm{c}}$ ranging 


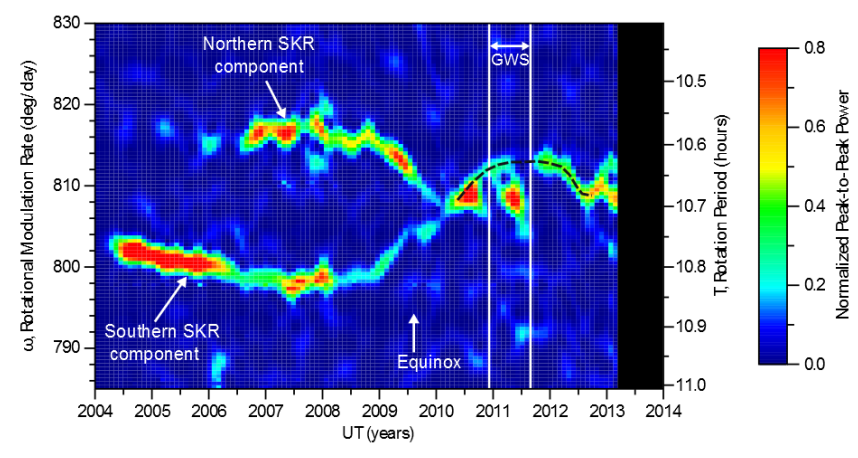

Figure 1. Normalized peak-to-peak power of Saturn kilometric radiation modulation as a function of time from 2004 until early 2013. The ordinate shows the angular velocity (rotation rate) in degrees per Earth day (left side) and the corresponding rotation period in hours (right side). The normalized peak-to-peak power is given by the color bar, and the signal processing was done as in Gurnett et al. (2009). The duration of the Great White Spot (GWS) event is indicated. There are two periods related to SKR from different hemispheres until shortly after equinox, but there is mainly one period until 2013 (black dashed line) except for a few months in early 2011.

$-1.1<d_{\mathrm{c}}<-0.5$ (right-hand polarization), whereas for the southern SKR the criteria were SNR $>10$ and $0.5>d_{\mathrm{c}}>1.1$ (left-hand polarization). The absolute value of the circular polarization $\left|d_{\mathrm{c}}\right|$ can go beyond its theoretical maximum of 1 due to measurement errors of about $10 \%$. This is a somewhat simpler separation algorithm compared to the one used by Fischer et al. (2014), who also included the spacecraft latitude and took the incoherent superposition of northern and southern SKR into account. However, the results are very similar. After this separation the SKR intensities of both hemispheres are integrated separately, and then the tracking filter analysis described above is applied to both of them.

Until early 2010 the SKR modulation spectrogram in Fig. 1 clearly shows two components. The stronger one has a period of $\sim 10.8 \mathrm{~h}$ from 2004 until 2009, and it is attributed to SKR originating from the southern hemisphere (Gurnett et al., 2009). The second component related to northern SKR has a shorter period of $\sim 10.6 \mathrm{~h}$ (note that both periods should be present in the complete SKR signal). From 2009 forward, both periods seem to converge until they become approximately equal in early 2010 . Here we focus on the time after spring 2010 and do not discuss when and if the periods crossed. The northern and southern SKR period cannot be distinguished in the large spectral feature present in the Fig. 1 modulation spectrogram during most of the year 2010. With the exception of spring 2011, it looks like only one SKR period is present in Fig. 1 until early 2013. The SKR rotation rate rises to about $812^{\circ} \mathrm{day}^{-1}(10.64 \mathrm{~h})$ at the end of 2010 . Then there is a sharp decrease in rotation rate, and one might have expected a course as indicated by the black dashed line. However, the rotational speed goes down by $\sim 0.5 \%$ from

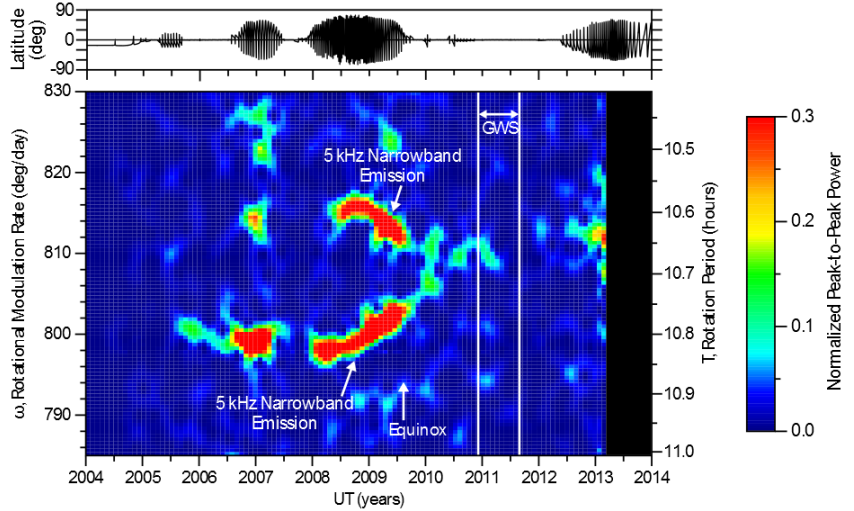

Figure 2. Normalized peak-to-peak power of Saturn narrowband radio emission modulation. The display is similar to Fig. 1, and the upper panel shows the planetocentric latitude of Cassini as a function of time. Narrowband emissions at $5 \mathrm{kHz}$ are more frequently observed from high latitudes, leading to strong modulation signals during Cassini's high-latitude orbits. The modulation rate shows a kink at the end of 2010 when the Great White Spot (GWS) event starts and a decreasing rate afterwards. Similar to SKR in Fig. 1, there are two components until 2010, but there is only one afterwards until early 2013.

812 to $808^{\circ}$ day $^{-1}$ (the broad signal even extends down to $805^{\circ}$ day $^{-1}$ in July 2011), followed by a large discontinuity and a sudden jump back to a faster rotation. This large jump discontinuity is a unique feature which is not present anywhere else in Fig. 1. The start and end of this rather atypical SKR behavior coincides roughly with the occurrence of the Great White Spot in Saturn's atmosphere (Sánchez-Lavega et al., 2011, 2012; Fischer et al., 2011; Sayanagi et al., 2013), whose temporal occurrence is indicated by the vertical white lines in Fig. 1.

The modulation spectrogram of Saturn narrowband radio emissions from 2004 until early 2013 is shown in Fig. 2. It looks relatively similar to the SKR modulation of Fig. 1 with two periods until the end of 2009, which are similar to the SKR periods as shown by Ye et al. (2010b). The narrowband radio emission modulation signal is clearly weaker during the Cassini equatorial orbits, as can be seen by looking at the top panel in Fig. 2, which gives the latitude of the Cassini spacecraft. Ye et al. (2010a) have shown that the $5 \mathrm{kHz}$ Saturn narrowband emissions are more intense when observed from higher latitudes. The modulation signal is very broad in early 2010 extending from 805 to $813^{\circ}$ day $^{-1}$. Afterwards there is a decrease in rotation rate from the end of 2010 until spring 2011 starting approximately with the GWS event, which is indicated by vertical white lines similar to Fig. 1. Then the narrowband emission modulation signal is almost absent from spring 2011 until mid-2012.

In the upper panel of Fig. 2 we have plotted the planetocentric latitude of the Cassini spacecraft. It can be seen that from autumn 2009 until mid-2012 Cassini is mainly located 


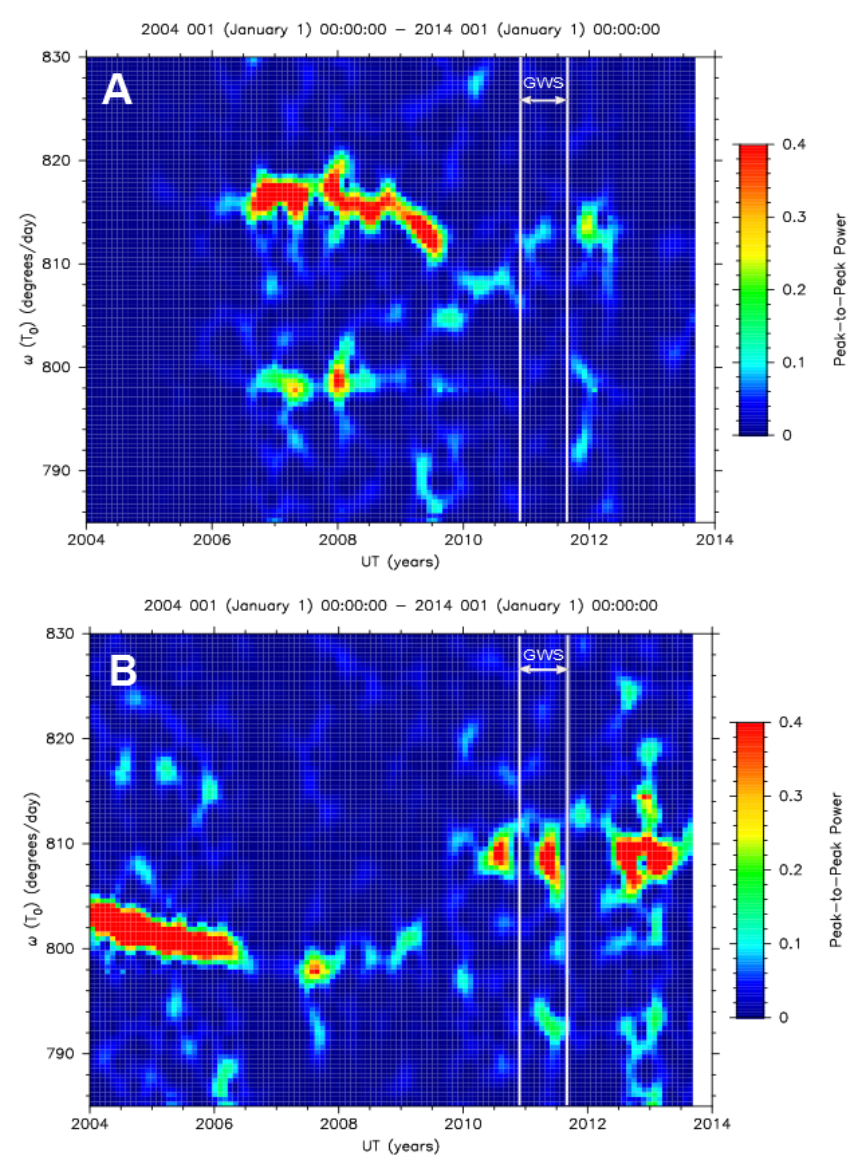

Figure 3. Normalized peak-to-peak power of northern and southern SKR modulation as a function of time from 2004 until mid-2013. The upper panel (a) shows the periodogram for the northern SKR and the lower panel (b) for the southern SKR. The display is similar to Fig. 1, and the vertical white lines indicate the start and end of the GWS event.

in the equatorial plane. In contrast to narrowband radio emissions, the equatorial orbits are advantageous for the simultaneous detection of SKR from both hemispheres. Beyond the equatorial shadow (from $\sim 4 R_{\mathrm{S}}$ inward, Lamy et al., 2008b), SKR originating from both the northern and southern hemisphere can almost always be observed. This has led to the strong SKR modulation signal after equinox in Fig. 1. The first two high-latitude excursions of Cassini in 2006/2007 and from early 2008 until mid-2009 were characterized by long passages of Cassini through the northern hemisphere with only short dips into the southern hemisphere. This has led to a weaker SKR modulation signal for the southern hemisphere during those periods (see Fig. 1) since southern SKR is much less likely to be observed from the northern hemisphere.

In Fig. 3 two modulation spectra are shown, one for the northern right-hand polarized SKR in the upper panel a, and one for the southern left-hand polarized SKR in the lower panel b. Fig. 3 shows again that before Saturn equinox, the northern SKR had a period of $\sim 10.6 \mathrm{~h}$ (or $\sim 815^{\circ} \mathrm{day}^{-1}$ ), and at the same time, the southern SKR period was $\sim 10.8 \mathrm{~h}$ (or $\sim 800^{\circ} \mathrm{day}^{-1}$ ) (Gurnett et al., 2009). The absence of the northern modulation signal before 2006 can partly be explained by Cassini's southern latitude (see top panel of Fig. 2) and by the low northern SKR intensities during southern summer conditions as shown by Kimura et al. (2013). The southern period of $\sim 10.8 \mathrm{~h}$ can also be discerned in the northern SKR spectrogram, especially from the end of 2006 until mid-2008. Lamy (2011) suggested that the secondary peak at $\sim 10.8 \mathrm{~h}$ in the northern signal is likely to reflect a real physical dual modulation. He argues that SKR sources have been identified on closed field lines and that auroral electrons accelerated in one hemisphere can ultimately reach the other one. However, Fischer et al. (2014) argue that secondary signals can also be caused by amplitude modulation with the Cassini orbital period, by the separation with regard to wave polarization, or by searchlight-like SKR components. The northern SKR modulation signal has only modest intensity from the end of 2009 until the end of 2011 (the first 2 years after equinox). Starting in 2010, the major strong modulation signals of SKR come from the southern hemisphere with one large and intense spectral feature in each year from 2010 to 2012/2013. During the GWS event, marked by vertical white lines in Fig. 3, the southern SKR signal is much stronger than the northern one. The northern SKR starts with a rotation rate around $812^{\circ}$ day $^{-1}$ in early 2011 and rises to about $814^{\circ} \mathrm{day}^{-1}$ in May/June 2011. The southern SKR shows a very broad $\left( \pm 3^{\circ}\right.$ day $\left.^{-1}\right)$ and intense signal around $808^{\circ}$ day $^{-1}$ in spring/early summer 2011. At the end of 2010/early 2011 the signal is much weaker and at a rate of $812^{\circ} \mathrm{day}^{-1}$, similar to the northern SKR rate at that time. After mid-2012 until autumn 2013, Fig. 3a shows that the northern SKR modulation signal is absent due to Cassini's high-latitude orbits with long dwell times in the southern hemisphere (see top panel of Fig. 2).

\section{Detailed behavior of SKR modulation during the GWS event}

Lightning activity measured by the RPWS instrument started on 5 December 2010 and lasted until 28 August 2011 as indicated by the vertical white lines in Figs. 1 to 3 . The giant thunderstorm raged at a kronocentric latitude of $\sim 35^{\circ}$ north (all latitudes in this paper are planetocentric), and a convective region of $\sim 10000 \mathrm{~km}$ in latitudinal diameter (called the storm's head) showed a drift of $2.8^{\circ}$ day $^{-1}$ to the west (Sánchez-Lavega et al., 2011, 2012; Fischer et al., 2011; Sayanagi et al., 2013) with respect to the Voyager Saturn Longitude System. This corresponds to a rotation rate of $808^{\circ}$ day $^{-1}$ or a period of $10.693 \mathrm{~h}$. Thunderclouds in the upper troposphere drifted away with the zonal wind flow creating a turbulent tail which encircled the whole planet within a few weeks after the start of the storm. Figure 4 


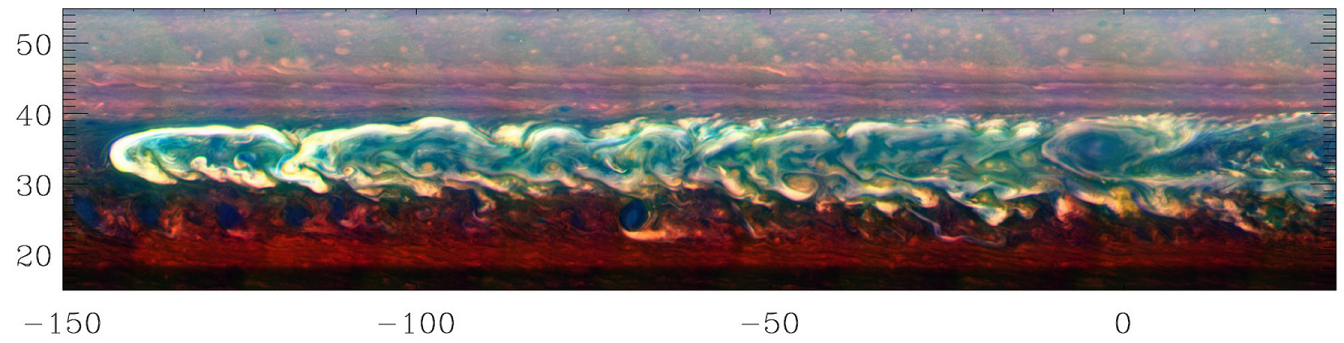

Figure 4. Contrast-enhanced mosaic image of Saturn's Great White Spot. The images were taken by Cassini ISS (Imaging Science Subsystem) on 6 March 2011. One can clearly see the head of the storm on the left and an anticyclonic vortex in the tail on the right side around 0 eastern longitude. The mosaic covers an area from 15 to $55^{\circ}$ north planetocentric latitude, and a longitude range of $180^{\circ}$. Image by NASA/JPL/SSI.

shows an image of the main part of the storm taken in early March 2011 by the Cassini camera. Lightning flash rates were extraordinarily high from mid-December to mid-July, indicating violent vertical moist convection, and the main thunderstorm cell was in the GWS head region (Sayanagi et al., 2013), although some lightning was also present in the tail region (Dyudina et al., 2013). The GWS also had a significant influence on the temperature and dynamics of Saturn's stratosphere (Fletcher et al., 2011, 2012). Two distinct warm airmasses in Saturn's stratosphere, located near $0.5 \mathrm{mbar}$ around the GWS at $35^{\circ}$ north latitude, formed and intensified from January until April 2011, before they merged into one large hot spot in late April with a temperature $80 \mathrm{~K}$ higher than the quiescent surroundings (Fletcher et al., 2012).

The tracking filter analysis of Gurnett et al. (2009, 2010, 2011) employed in the last section uses sliding windows of 240 days and time steps of 30 days. To gain further insight into the SKR modulation during the GWS event, we simply follow the SKR phase as a function of time allowing us to see changes on shorter timescales. Figure 5 shows the phases of northern and southern SKR, displayed with respect to the constant guide period of $10.6567 \mathrm{~h}$ in a time interval of 1 year starting on 15 October 2010. The constant guide period of $10.6567 \mathrm{~h}$ is arbitrarily chosen as identical to the Voyager radio period of Desch and Kaiser (1981). There is no artificial phase shift introduced between northern and southern SKR phase, and the phase is set to zero at 00:00 SCET on 31 December 2003, i.e., we use DOY (day of year) 2004 as our time counter. Figure 5a shows the normalized intensity of northern SKR as a function of time and phase, and Fig. 5b shows the same for southern SKR. Similar plots have been made by Kurth et al. $(2007,2008)$ for the complete SKR signal, but here we have separated the SKR by polarization as described in the beginning of the last section. Such phase plots have also been drawn in Fischer et al. (2014) for the 4 years from 2009 to 2012, but here we focus on the time around the GWS event. In Fig. 5 the phase is plotted over two rotations $\left(720^{\circ}\right)$ to enable a better tracking of the SKR intensity maxima. The white and red lines track those maxima for northern and southern SKR phases, respectively. They were drawn by eye with one data point every 10 days, taking into account the SKR intensity maximum values at each rotation and the general trend. Furthermore, a directional statistics method was employed (for details see Appendix C of Fischer et al., 2014) to determine the SKR maxima in a quantitative way, and one can see in Fig. 5 that the magenta stars (from this method) follow closely the white and red lines. The northern SKR phase has a positive slope from the start of the plot on day 2480 to about the beginning of the GWS event (day 2531) and for a few days around day 2750. This means that the period $\tau_{n}$ is larger (slower) than the constant guide period, i.e., $\tau_{n}>10.6567 \mathrm{~h}$. For the positive slope, the guide phase is increasing with time, and therefore it is analogous to a westward longitude system where, seen from a fixed position, the longitude is increasing with time. This is analogous to a westward drift or a slower rotation period since Saturn rotates eastward. For the first 2-3 months of the GWS event, the slope of the white line in Fig. 5a is zero on average, and the slope of the red line in Fig. 5b is only slightly negative. This means that here the northern and southern SKR periods are almost equal to the constant guide period of $10.6567 \mathrm{~h}$ (or $\sim 810.76^{\circ}$ day $^{-1}$ ). The negative slope of the white line from about day 2610 to the end of the plot (with the exception of around day 2750) indicates a northern SKR period that is smaller (faster) than the guide period, i.e., $\tau_{n}<10.6567 \mathrm{~h}$. A decreasing guide phase difference (negative slope) can then be understood as a decreasing western longitude or an eastward drift which means a faster rotation. The southern SKR phase maximum (red line) has a significant change from positive to negative slopes shortly before the end of the GWS event (28 August).

From the slope of the SKR phase, the rotation rate can be derived. The exact mathematical relation between the phase $\phi(t)$ of the SKR maxima in Fig. 5 and the rotation rate $\omega(t)$ is the following (see Appendix $C$ of Fischer et al., 2014). With $\omega_{\mathrm{g}}$ being the guide rotation rate $\left(\sim 810.76^{\circ} \mathrm{day}^{-1}\right), \omega(t)$ can be calculated with the relation $\omega(t)=\omega_{\mathrm{g}}-\mathrm{d} \phi(t) / \mathrm{d} t$ with the last term $\mathrm{d} \phi(t) / \mathrm{d} t$ being the slope of the white and red lines in Fig. 5 tracing the SKR maxima. This mathematical procedure leads us to Fig. 6, where we have drawn the rotation 

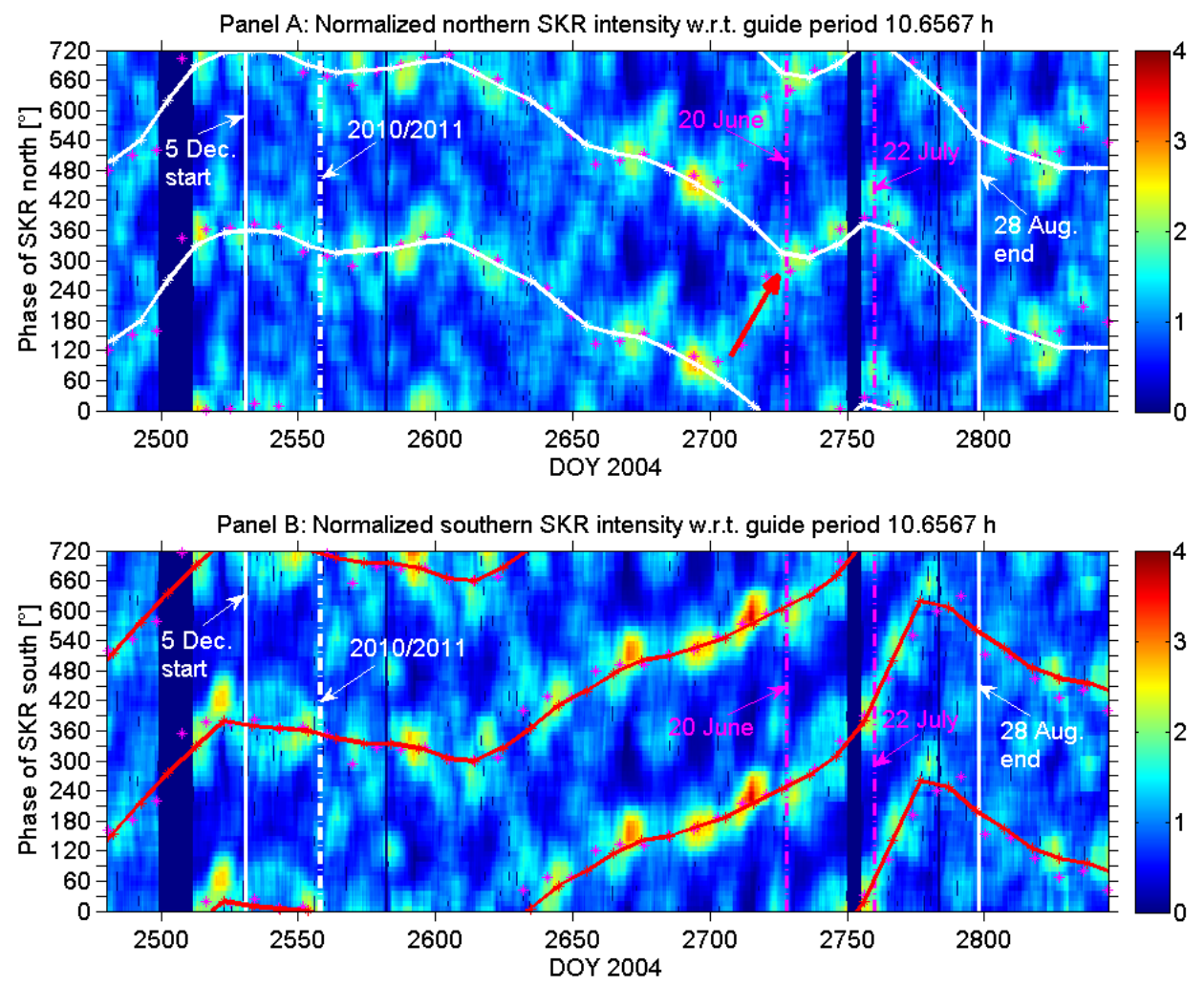

Figure 5. Phase drift of northern and southern SKR with respect to a constant guide period of $10.6567 \mathrm{~h}$ around the GWS event. We show the time interval from day 2480 to 2845 which lasts 1 year starting on 15 October 2010 . The SKR intensities are averaged for guide phase intervals of $10^{\circ}$ (corresponding to $\sim 18 \mathrm{~min}$ ), and they are plotted over two rotations $\left(720^{\circ}\right)$. The intensities (north and south) are normalized by dividing them by the averaged SKR intensity over one rotation, and this ratio $r$ is color-coded according to the color bar on the right side. To enhance the visibility of the SKR maxima, the whole 2-D matrix of SKR intensity ratios ( $r$ as a function of time and phase) was smoothed by replacing each value $r$ of the intensity ratio by the mean over a rectangle of $70^{\circ}$ and 17 rotations centered on $r$. The white and red lines trace the northern and southern SKR maximum values, respectively. The vertical white lines mark the start of the lightning activity related to the GWS event on 5 December 2010, and the last day with lightning activity on 28 August 2011. The dash-dotted vertical magenta lines inside those boundaries are placed on 20 June and 22 July 2011. Those dates are related to pronounced changes in lightning activity which is described in more detail in the text. The red arrow in (a) is also discussed in the text. The white dash-dotted line indicates the turn of the year 2010/2011. The magenta stars were calculated with directional statistics (see Appendix C of Fischer et al., 2014) to help tracing the SKR maxima.

rates of northern and southern SKR as a function of time over 2.5 years from the beginning of 2010 until mid-2012. Figure 6 shows that northern and southern SKR periods are similar for extended intervals of time. This is the case for a first interval starting in March 2010 and lasting for almost 1 year until February 2011, and for a second interval from the end of August 2011 to June 2012. The time interval of the GWS event is indicated in Fig. 6 as well, and here the periods of northern and southern SKR are largely different except for the first 2-3 months. The horizontal dotted line in Fig. 6 indicates the guide period of $10.6567 \mathrm{~h}$. Periods longer than this guide period (smaller rotation rates) have positive slopes in the SKR phase plot, whereas periods smaller than the guide period (larger rotation rates) have negative slopes in the SKR phase plot of Fig. 5.
We now discuss the course of the SKR phases during the GWS event in more detail. Similar to previous figures, we set 5 December 2010 (day 2531) as the start date and $28 \mathrm{Au}-$ gust 2011 (day 2797) as the final date of the GWS event, indicated by the vertical white lines in Fig. 5 and by the black dashed lines in Fig. 6. At first we see a kink in the phase of northern and southern SKR (white and red lines tracing the maxima) roughly co-located with the start of the GWS event. This is associated with an increase in rotation rate from $\sim 805$ to $\sim 813^{\circ} \mathrm{day}^{-1}$. These changes might not be related to the GWS event since the kink seems to be slightly earlier and similar changes in rotation can be seen throughout the year 2010 as well. From the end of 2010 until February 2011, the SKR modulation signal in Fig. 1 has modest intensity, it gets broader, and there seems to be little change in the rotation period. This can also be seen in Fig. 6, where northern 


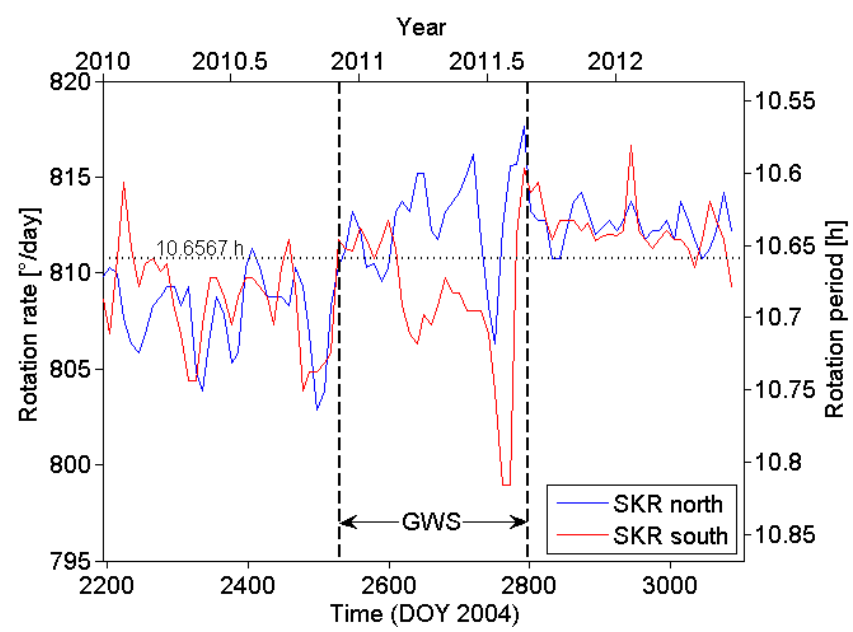

Figure 6. Rotation rates/periods of northern and southern SKR over 2.5 years from the beginning of 2010 until the middle of 2012. The SKR rates have been derived by following the SKR phases (Fischer et al., 2014). They are displayed as solid lines in blue and red color for northern and southern SKR, respectively. The lower horizontal axis denotes the time in days of year 2004, and the upper horizontal axis denotes the years. The left vertical axis shows the rotation rate in degrees per day, whereas on the right one can see the rotation period in hours. The horizontal dotted black line indicates the constant guide period of $10.6567 \mathrm{~h}\left(810.7576^{\circ}\right.$ day $\left.^{-1}\right)$. The black dashed lines indicate the start and end of the GWS event (5 December 2010 until 28 August 2011), respectively.

and southern periods are at first similar, but later start to deviate from each other around day 2600 (mid-February 2011). Additionally, a careful inspection of Fig. 5 shows that northern and southern SKR phase are very similar before they start to deviate from each other also shortly after day 2600.

To illustrate all this more clearly we have drawn the phase difference and the rotation rate difference between northern and southern SKR as a function of time from the beginning of 2010 until the middle of 2012 in Fig. 7. One can clearly see in this figure that the time interval of the GWS event is special. It is mainly during this time that the SKR periods and phases show significant hemispherical differences (except for the first 2-3 months). There are also some differences in early 2010 going back to the large hemispherical differences between northern and southern period before equinox. By March 2010 the blue line in Fig. 7 indicating the phase difference finally arrives around zero which indicates that northern and southern SKR phases are locked. The phases stay locked until February 2011, and they get locked again right after the end of the GWS event around day 2800 . After that, the phase difference is $-1080^{\circ}$, which corresponds to $0^{\circ}$ (locked phases) taken modulo $360^{\circ}$, and it stays like that until the middle of 2012. Similarly, the difference in SKR rotation rates between the northern and southern hemisphere is most pronounced during the GWS event.

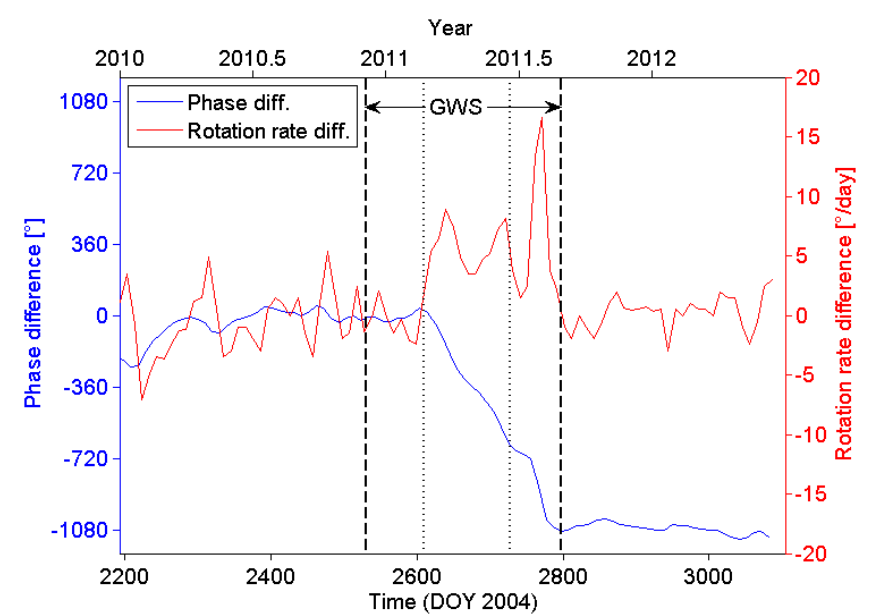

Figure 7. Phase difference and rotation rate difference of northern to southern SKR as a function of time over 2.5 years from the beginning of 2010 until the middle of 2012. The SKR phase difference (northern phase minus southern phase) is plotted as a blue line and refers to the left (blue) vertical axis. The SKR rotation rate difference (northern minus southern rate) is plotted as a red line and refers to the right (red) vertical axis. The time is given in days of year 2004 at the bottom horizontal axis and in years at the top horizontal axis. The time of the Great White Spot (GWS) event lasting from 5 December 2010 to 28 August 2011 is indicated. A vertical dotted line was inserted on day 2610 (22 February 2011) to indicate the starting SKR phase and rotation rate differences, and another one was inserted on day 2728 (20 June 2011) marking the date of the GWS head-vortex collision, which resulted in reduced convective activity.

Under the assumption that there is a relation between GWS and SKR modulation, Figs. 5 to 7 show that it took some time (2-3 months) before the GWS could exert its influence. This could be due to the fact that the storm first had to grow to a certain size to affect the SKR. The lightning activity started in a small spot on 5 December 2010, which quickly grew and reached a diameter of about $10000 \mathrm{~km}$ about 3 weeks after it started. Although the clouds quickly drifted eastward (with about 5-6 ${ }^{\circ}$ day $^{-1}$ ) and formed the long tail driven by the zonal wind, the extension of the convective activity from the GWS head into the tail region was considerably slower $\left(\sim 1^{\circ} \mathrm{day}^{-1}\right)$. This progressive extension was observed by an increasing longitudinal range of emitted lightning radio emissions (Fischer et al., 2011; and Fig. 9 in Sayanagi et al., 2013), and lightning is known as a good indicator of vertical convection. When the hemispherical deviation of SKR phases and periods started to become large in March 2011, the thunderstorm cells with lightning activity were present over about a quarter of Saturn's circumference at $35^{\circ}$ north extending eastward from the storm's head. At the time of the GWS image in Fig. 4, the source regions of lightning were roughly located in the eastern longitude range of -140 to $-50^{\circ}$, whereas the whole cloudy tail 
was much more elongated. We also note that the effect of the GWS on Saturn's stratosphere (Fletcher et al., 2011, 2012) was also not present immediately after the start of the lightning activity in early December 2010, but built up within the course of a few months.

From March until July 2011, the complete SKR modulation signal in Fig. 1 has its strongest power around an average rotation rate of $808^{\circ}$ day $^{-1}$ corresponding to a period of $10.69 \mathrm{~h}$. Figure 3 shows that this strong signal is mainly due to the southern SKR, and in Fig. 5b one can see the southern SKR with a positive slope of around $\sim 2.8^{\circ}$ day $^{-1}$ corresponding to a rotation rate of $808^{\circ}$ day $^{-1}$. We see a change in phase over about one rotation $\left(360^{\circ}\right)$ in the 130 days from days 2625 to 2755 . The phase of northern SKR started to deviate from the phase of southern SKR soon after day 2600, and we see a negative slope of the white line for the rest of the time displayed in Fig. 5a except for some days around day 2750 . One can actually see some signals roughly following the southern SKR phase as is indicated by the red arrow. These signals are co-located in phase with the strongest signals from the southern hemisphere in Fig. 5b, i.e., they could be due to electrons coming from the southern hemisphere as suggested by Lamy (2011). The same might be true for the northern signals around day 2750 where the SKR maxima shortly follow a line with a positive slope. We did not follow the path indicated by the red arrow since no corresponding modulation signals can be seen in the tracking filter analysis of Fig. 3a. However, the northern SKR phase maxima are very clear in the interval around day 2750 when the white line has a positive slope. This leads to almost similar periods of southern and northern SKR for a very short time around day 2750 as can be seen in Fig. 6. Such short-term variations can also be seen at other instances of time in Fig. 6, and they could be due to the influence of the solar wind (Zarka et al., 2007). They are absent in Figs. 1 and 3 due to the large window of 240 days used in the tracking filter analysis. The average rotation rate of $808^{\circ}$ day $^{-1}$ (or $10.693 \mathrm{~h}$ ) of the southern SKR is suspiciously similar to the rotation rate of the GWS head. This may be no coincidence, and it has been speculated that atmospheric features can impose their rotation period on the upper atmosphere and magnetosphere (Smith, 2006). It seems strange that there could be an influence of the GWS located in the northern hemisphere on the SKR from the southern hemisphere, and we will discuss this in the next two sections. Interestingly, the magnitude of the slope of the northern SKR phase is similar to the one of the southern SKR phase, only the sign is different. We can see a negative slope of about one rotation $\left(360^{\circ}\right)$ within the 130 days from day 2600 to 2730 , i.e., $-2.8^{\circ}$ day $^{-1}$. The narrowband radio emission modulation displayed in Fig. 2 seems to largely follow the behavior of the southern SKR during the GWS event. The modulation signal in Fig. 2 is broad, and the rotation rate started to go down roughly when the giant thunderstorm started, and it also came close to $808^{\circ}$ day $^{-1}$ in spring 2011. The signal disappears in May 2011, most likely due to the low detection probability of narrowband radio emissions from low latitudes (Ye et al., 2010a).

The final 2-3 months of the GWS event are also an interesting time interval. Two dates (20 June and 22 July) are important as they are related to pronounced changes in the lightning activity. A plot showing the lightning flash rates from mid-June to the end of August 2011 can be found in Fig. 10 of Sayanagi et al. (2013). First, the large anticyclonic vortex and the head of the GWS collided in midJune 2011 (Sayanagi et al., 2013; Sánchez-Lavega et al., 2012). The large anticyclonic vortex had already developed in late December 2010, and it drifted at $0.85^{\circ} \mathrm{day}^{-1}$ to the west (Sayanagi et al., 2013). Since the storm's head was moving at $2.8^{\circ}$ day $^{-1}$ westward, the anticyclonic vortex is located eastward of the head in the storm's tail region in our Fig. 4 (around $0^{\circ}$ eastern longitude). After the head-vortex collision there was a significant decrease in lightning flash rate on 20 June 2011. However, the lightning activity recovered to a high level, but it became variable with maxima around 1 and 10 July, and minima around 7 and 15 July (see Fig. 10 of Sayanagi et al., 2013). After 22 July the lightning activity became much weaker and similar to the smaller $2000 \mathrm{~km}$ sized storms that were observed prior to the GWS in the southern hemisphere (Fischer et al., 2008). Typical flash rates for those smaller storms are a few flashes per minute, whereas the typical GWS flash rate was higher than 10 flashes per second (Dyudina et al., 2013; Fischer et al., 2011). So, after 22 July the lightning activity became much weaker and intermittent until it finally disappeared after 28 August 2011. We have drawn two vertical dash-dotted magenta lines in Fig. 5, the first one at 20 June (day 2728), and the second one at 22 July (day 2760) before the vertical white line on 28 August (day 2797) indicating the end of lightning activity. One can see some changes in the SKR phase drift plot of Fig. 5 related to those dates. The slopes of the northern and southern SKR are both positive between the dash-dotted magenta lines in Fig. 5. In particular, the short time interval with positive slope of the northern SKR phase starts and ends around those two dates marked by the dash-dotted magenta lines. The slope of the red line indicating the southern SKR phase shows an increase between 20 June and 22 July. About 2 weeks after 22 July there is a kink in the southern SKR phase with a change from a positive to a negative slope. The average slope of the southern SKR phase after 20 June is $\sim 9^{\circ}$ day $^{-1}\left(\sim 360^{\circ}\right.$ in $\sim 40$ days) which corresponds to a rotation rate of $\sim 802^{\circ}$ day $^{-1}$. In Fig. 6 one can see that the rotation rate goes down to $798^{\circ}$ day $^{-1}$, i.e., the maximum slope of the southern SKR phase is $\sim 13^{\circ}$ day $^{-1}$. Figure $3 \mathrm{~b}$ shows a weak modulation signal of southern SKR centered at $800^{\circ}$ day $^{-1}$ in the last 2-3 months of the GWS event. Hence, it is likely that this spot is related to the quick change in southern SKR phase during that time. (The more pronounced spot around $792^{\circ}$ day $^{-1}$ in 2011 in the southern SKR modulation spectrum of Fig. 3 is interpreted by Fischer et al. (2014) as being due to amplitude modulation by 
the Cassini orbital period or due to a searchlight-like SKR component.) The significant kink in the slope of the southern SKR phase in mid-August 2011 leads to the large jump in rotation rate, up to $813^{\circ}$ day $^{-1}$. Around day 2800 (end of August), the quick change in rotation rate can also be seen in Figs. 1 and 3 leading to the large jump discontinuity of the SKR modulation signal. Figures 6 and 7 show that northern and southern rotation rates were again very close in late $\mathrm{Au}-$ gust 2011, similar to the situation before March 2011. Similarly, Figs. 5 and 7 show us that the phases of northern and southern SKR reached similar values in late August 2011.

\section{On a possible physical relation of the GWS to the SKR periodicity}

The obvious question is how a thunderstorm located in Saturn's troposphere around $35^{\circ}$ north can influence the auroral latitudes around $75^{\circ}$ which are the magnetic footprint latitudes of SKR and narrowband radio emission sources. It is very unlikely that the GWS redistributed so much mass to cause a substantial change in Saturn's large moment of inertia of $C=4.3 \times 10^{41} \mathrm{~kg} \mathrm{~m}^{-2}$ (Helled et al., 2009). The mass of the thundercloud can be estimated as $3 \times 10^{19} \mathrm{~kg}$ from its "thickness" of 1 bar $\left(\sim 10^{4} \mathrm{~kg} \mathrm{~m}^{-2}\right)$ and its dimension in latitude $(10000 \mathrm{~km})$ and longitude $(300000 \mathrm{~km})$ (SánchezLavega et al., 2011; Fischer et al., 2011). If one adds this mass at Saturn's mean radius of $R=58255 \mathrm{~km}$, the relative increase in moment of inertia and thus the relative decrease in rotation rate would only be $0.00002 \%$. A number of authors have speculated that Saturn's equatorial wind speed was reduced by the equatorial GWS of 1990. A $20 \%$ change in equatorial wind speed could be related to a change of the internal rotation rate by $0.1 \%$ (Sayanagi and Showman, 2007; Heimpel and Aurnou, 2012). However, observations during and after the 2010/2011 GWS (Sánchez-Lavega et al., 2012; Sayanagi et al., 2013) have shown only minor changes in Saturn's tropospheric zonal wind speeds.

The GWS did substantially alter the atmospheric circulation at higher altitudes; it modified stratospheric zonal jets, and generated hot beacons (which merged into one hot spot in spring 2011) of infrared radiation with large longitudinal temperature contrasts (Fletcher et al., 2011, 2012). Hence, there is observational evidence that the tropospheric storm cell produced effects that penetrated hundreds of kilometers (up to the $1 \mathrm{mbar}$ level) into Saturn's stratosphere. In contrast to that, smaller $2000 \mathrm{~km}$ sized thunderstorms did not produce changes in temperature and chemical composition in the stratosphere (Hurley et al., 2012). These smaller thunderstorms were mostly observed at a latitude of $35^{\circ}$ south (Fischer et al., 2008), and they seem to have no effect on the SKR period. The vigorous convection of the GWS should have excited a broad spectrum of wave activity (Fletcher et al., 2012) by which energy and angular momentum should have been transported not only up to the stratosphere, but also way beyond, up to thermospheric altitudes.

On Earth, penetrative convection at the tropopause during severe thunderstorms is a well-known generation mechanism of gravity waves (Pierce and Coroniti, 1966). The waves can transport energy in the vertical direction as well as horizontally below the tropopause. It has been observed that the horizontal waves can trigger additional thunderstorm cells (Balachandran, 1980). This process might have been at work also on Saturn, where thunderstorm cells were also observed in the GWS tail region (Sayanagi et al., 2013; Dyudina et al., 2013). Features resembling a horizontal gravity wave packet with a wavelength around $200 \mathrm{~km}$ were spotted at the westernmost front of the GWS head (García-Melendo et al., 2013). Gravity waves emitted by the GWS might have large power. Using a vertical energy flux of $0.1 \mathrm{~W} \mathrm{~m}^{-2}$ in the form of gravity waves as found for terrestrial thunderstorms (Stull, 1976), an area the size of the GWS head $(10000 \mathrm{~km} \times 10000 \mathrm{~km})$ would produce $10^{13} \mathrm{~W}=10 \mathrm{TW}$. Additional storm cells in the tail would lead to a further increase of that power. The power of the whole thunderstorm was comparable to Saturn's total radiated power (Fischer et al., 2011; Dyudina et al., 2013) of the order of $\sim 10^{17} \mathrm{~W}$, meaning that a fraction around $10^{-4}$ of the thunderstorm's power might be converted into vertical gravity wave power.

The dynamics of Saturn's thermosphere is largely unknown, and general circulation models (GCMs) are applied. One potential source of energy to reproduce the high thermospheric temperature of $420 \mathrm{~K}$ is heating by gravity waves (Joule heating by currents across the auroral oval is another important energy source). Taking a heating rate of $0.4 \mathrm{~mW} \mathrm{~m}^{-2}$ (Yelle and Miller, 2004) as found for Jupiter, gravity waves at Saturn would globally deposit a power of $\sim 18 \mathrm{TW}$ into the thermosphere (Müller-Wodarg et al., 2006 ), enough to heat up the thermosphere to $420 \mathrm{~K}$. If only a few percent of the large gravity wave power calculated above $(\sim 10 \mathrm{TW})$ also reaches the upper atmosphere, one can thus expect significant changes in the thermospheric temperatures and winds. Hence, energetically it should be possible that gravity waves created by the convective activity in a GWS exert a large influence on the thermosphere. GCMs at Saturn show meridional winds of $200-300 \mathrm{~m} \mathrm{~s}^{-1}$ in the upper atmosphere (Müller-Wodarg et al., 2006), so processes at lower latitudes might influence the dynamics at higher latitudes. Another option for thermospheric wave heating would be the dissipation of acoustic waves from thunderstorms as suggested for Jupiter (Schubert et al., 2003). An additional heat source like the GWS at mid-latitudes would lower the temperature gradient between the auroral and the equatorial region, leading to a decrease in high-latitude westward thermospheric winds according to the model of Müller-Wodarg et al. (2006). Their simulation S6 calculated under equinox conditions, including Joule heating but without gravity wave heating, shows a higher westward wind speed (i.e., slower rotation) at high latitudes than their simulation S7 including 
Joule heating and gravity wave heating (see their Table 1 and their Figs. 6 and 7). Hence, in this case the gravity wave heating would speed up the atmospheric rotation at high latitudes and as a consequence SKR would show a faster rotation (remember that we observed an increase of northern SKR rotation, but a slowdown of southern SKR rotation). Matcheva and Barrow (2012) suggest that gravity waves also exist in Saturn's upper atmosphere and that they cause a vertical layering of ions and electrons with a typical scale of $\sim 300 \mathrm{~km}$ as observed by the Cassini radio occultations.

The transfer of gravity wave energy to high altitudes can alternatively be discussed in terms of transfer of momentum. When atmospheric gravity waves dissipate, they transfer their momentum to the background flow of the atmosphere (Hines, 1974). Recent simulation results for Earth show that gravity waves from deep convection might lead to global changes in the background neutral winds of the thermosphere (Vadas and Liu, 2009). This means that not only the region close to the gravity wave source is influenced, but the large gravity wave power of the GWS might have had a global influence on Saturn's thermosphere. This could explain why a gravity wave source at mid-latitudes might also influence auroral latitudes. Interestingly, the mean dynamical effect of gravity waves is the deceleration of the mean zonal flow, and the gravity wave drag might be comparable to the ion drag (Yiğit et al., 2012). The thermospheric horizontal body force is usually directed against the background wind flow (Vadas and Liu, 2009). Since the GWS as the source of the gravity waves initially had a smaller rotation rate than the southern SKR it might have decelerated the neutral winds that drag the hypothetical ionospheric vortex of Jia et al. (2012) around Saturn's auroral oval. The decelerating mechanism of gravity wave momentum deposition could indeed even explain the adjustment of the rotation period of southern SKR and the GWS head, which had the same period of $\sim 10.69 \mathrm{~h}$ over several months in 2011 .

The large change of the SKR period in August 2011 could also be explained by the reduction and cessation of the convective activity. The short radiative time constant in Saturn's upper atmosphere (where the ionospheric Pedersen currents flow) should lead to a quick loss in energy and momentum as soon as significantly less or no more gravity wave energy and momentum is resupplied from below. The radiative time constant allows the estimation of how long it should take for a temperature perturbation to relax, and it depends largely on the altitude (it is about 25 years for Saturn's troposphere). We did not find the radiative time constant of Saturn's thermosphere in the literature, but for Jupiter it was estimated as $\tau_{\text {rad }} \approx 4 \times 10^{6} \mathrm{~s} \approx 46$ days (Yelle and Miller, 2004). Due to the similarity of gas giant atmospheres, this value should be of the same order of magnitude in Saturn's thermosphere (I. C. F. Müller-Wodarg, personal communication, 2014). Hence, it should have lasted several weeks or a few months for the disturbance of the GWS to disappear, consistent with our description of the final 2-3 months of the
GWS event in the last section. After the head-vortex collision of mid-June 2011 (first magenta dashed line in Fig. 5) the convective activity was already reduced, and after 22 July (second dashed line in Fig. 5) the convective activity was similar to smaller $2000 \mathrm{~km}$ sized thunderstorms. The smaller storms show no influence on the stratosphere according to Hurley et al. (2012), which suggests that their gravity waves are not energetic enough to have an influence on and above the stratosphere. The above-mentioned dates are weeks to months earlier than the jump of the SKR period at the end of August 2011. Similarly, the progressive growths of the GWS and the thermal inertia of Saturn's atmosphere might explain why it also took 2-3 months from December 2010 to late February/March 2011 until the GWS event started to influence the SKR periods and phases.

In Sect. 3 and in Fig. 6 we have shown that the northern SKR speeded up during the GWS event, whereas the southern SKR slowed down. It seems that the GWS has introduced a hemispherical asymmetry leading to different SKR rotation rates and phases in the north and south. The GWS was in the northern hemisphere, and so it is conceivable that the reduced temperature gradient between the northern auroral region and the GWS at northern mid-latitudes was the dominant effect that led to an acceleration of auroral thermospheric winds in eastward direction speeding up the northern SKR rate. For the southern hemisphere the reduction of mean zonal thermospheric wind by gravity wave drag might have been dominant, and so the southern SKR was slowed down to the same speed as the GWS head on average.

\section{On the difficulty of proving the SKR-GWS relation}

A temporal coincidence of two phenomena (special changes in SKR periods/phases and the GWS event) does not prove that there is a causal relation. However, there is a relation between Saturn's upper atmosphere and the magnetosphere via field-aligned currents, and many papers have suggested that the field-aligned currents might be driven by the upper atmosphere (Smith, 2006, 2011; Jia et al., 2012a; Jia and Kivelson, 2012). Oscillations close to the planetary period must be maintained in some manner by the internal rotation of the planet via a coupling through the atmospheric layers. Cowley and Provan (2013) have meticulously listed the periods of various features in Saturn's troposphere/stratosphere to compare planetary period oscillations in Saturn's magnetosphere with the periods of neutral atmospheric perturbations. However, most phenomena they list (North Polar Spot, North Polar Hexagon, String of Pearls, stratospheric infrared beacons) do not show sufficient vertical dynamics to be large sources of vertical gravity waves that might influence Saturn's atmosphere up to thermospheric altitudes. Even the $2000 \mathrm{~km}$ sized thunderstorms, which do clearly show vertical convection, are probably not energetic enough. As already mentioned, Hurley et al. (2012) found no changes in temperature 
and chemical composition in the stratosphere related to the $2000 \mathrm{~km}$ sized thunderstorms located at $35^{\circ}$ south. Therefore, no relation was found by Cowley and Provan (2013) between those phenomena and the planetary period oscillations in Saturn's magnetosphere. Regarding the GWS, their main argument against a relation is that the start and end of the GWS are not exactly contemporaneous with the jumps in period in the magnetic field data, i.e., they assume that there should be a prompt response of the upper atmosphere to processes in the troposphere. We argue that the GWS first had to grow to a certain size to exert its influence and that the atmosphere has a certain thermal inertia, which could be weeks to months in the regions of the thermosphere/ionosphere where the field-aligned currents flow. This delay time explains why the SKR periods started to change 2-3 months after the GWS event had started, and why the hemispherical period and phase deviations still continued for 2-3 months after the head-vortex collision of mid-June had significantly reduced the convective activity. We have illustrated this in Fig. 7 by drawing vertical dotted lines in late February 2011 and midJune 2011.

The dynamics of Saturn's upper atmosphere is a very complex problem, and maybe the hypothesis of a relation between the GWS and the SKR period could be reinforced by future modeling efforts. Currently, general circulation models (GCMs) from the troposphere up to the thermosphere/ionosphere do not yet exist for Saturn. The thermospheric GCM of Müller-Wodarg et al. (2006) starts $800 \mathrm{~km}$ above the 1 bar level. Other models like those of Friedson and Moses (2012) or Sayanagi and Showman (2007) are restricted to the troposphere and stratosphere. A good knowledge of the composition and all relevant physical processes (radiative transfer, eddy diffusion, photochemistry etc.) from Saturn's troposphere up to the thermosphere would be needed for such an advanced model that might emerge in the next years and is clearly beyond the scope of this paper. GCMs of the whole atmosphere exist for the Earth (e.g., Miyoshi and Fujiwara, 2008; Yiğit et al., 2012), and they do emphasize the importance of gravity waves created in the troposphere for thermospheric temperatures and winds.

The possible relation between the GWS and the SKR modulation is also hard to confirm experimentally by repeated measurements since GWSs are rare events that usually happen only once per Saturn year (29.5 Earth years). So far, six GWS events have been observed (1876, 1903, 1933, 1960, 1990, 2010/2011). It will be a very long time until we will have several cases where the observation of the GWS is also accompanied by SKR measurements. The latter cannot be done from the surface of the Earth due the low frequencies of SKR $(<1.2 \mathrm{MHz})$ which cannot penetrate the terrestrial ionosphere. Additionally, half of the six observed GWS events took place in the equatorial region $(1876,1933,1990)$ and had periods around 10.25 h (Sánchez-Lavega, 1994), and it is not clear if the SKR period can deviate that much from its mean period of $\sim 10.7 \mathrm{~h}$ or if an equatorial GWS could produce a significant hemispheric asymmetry in thermospheric wind speeds.

Finally, we note that we do not intend to explain the complete seasonal variation of the SKR modulation here. Great White Spots themselves are probably a seasonal phenomenon which tends to occur at northern summer conditions (Sánchez-Lavega, 1994), and the 2010/2011 GWS unexpectedly occurred just about 1.5 to 2 years after northern vernal equinox. However, they typically last for just a few months, which is only a small fraction of a full Saturn year. Other long-term processes like the seasonal variation of the Pedersen conductivity or the seasonal variation of the neutral wind speed in the auroral ionosphere might be relevant. In the years following the Saturn equinox of August 2009 one should expect similar conditions in the northern and southern hemisphere which should result in similar SKR periods and phases. Figures 6 and 7 show that the hemispheric periods and phases are indeed similar from spring 2010 until mid-2012 except for the time around the GWS. We suspect that the northern and southern SKR would have kept similar phases and periods in 2011 without the existence of the GWS, and that in this case the period might have followed the black dashed line in Fig. 1. The GWS probably only led to a temporary distortion of the normal seasonal conditions, and therefore northern and southern SKR periods and phases became similar again soon after the reduction and cessation of convective activity. One can expect that with the progression into northern summer the ionospheric conditions at both hemispheres will become different again, which should lead to a splitting of the SKR periods. The absence of the northern modulation signal until autumn 2013 does not allow us to tell if this splitting has already happened. Finally, there are no other long-lasting features (e.g., solar wind changes) other than the giant thunderstorm to coincide with the peculiar changes in SKR and narrowband radio emission modulation.

\section{Conclusions}

We have analyzed the rotational modulation and phases of Saturn kilometric radiation (SKR) and Saturn narrowband radio emissions specifically around the Great White Spot (GWS) event of 2010/2011. A tracking filter analysis technique was used to analyze the periodicities of the complete SKR signal, and of northern and southern SKR separated by wave polarization. We plotted the phase drift of normalized northern and southern SKR intensities versus time with respect to a constant guide period. We found that northern and southern SKR periods were very similar from spring 2010 until June 2012, except for March 2011 to August 2011 when northern and southern SKR periods and phases showed significant deviations from each other. We argue that these deviations could plausibly be due to the GWS, and we suggest that it is no coincidence that the visible head of the GWS had 
the same period of $\sim 10.69 \mathrm{~h}$ as the main southern SKR signal. We discussed a possible physical link via gravity waves that caused a global change in Saturn's thermospheric circulation by energy and momentum deposition. The giant thunderstorm on Saturn was most likely a large source of gravity waves with powers in the range of TW, which should have been enough power to exert a major influence on the planet's thermosphere. The GWS might have provided us a valuable hint that Saturn's upper atmosphere plays a crucial role for the planet's magnetospheric periodicities.

Acknowledgements. G. Fischer was supported by the Austrian Science Fund FWF (project P24325-N16) and by a short-term research scholarship at the University of Iowa in spring 2012. The research at the University of Iowa was supported by NASA through contract 1415150 with the Jet Propulsion Laboratory.

Topical Editor E. Roussos thanks J. Carbary and one anonymous referee for their help in evaluating this paper.

\section{References}

Andrews, D. J., Cecconi, B., Cowley, S. W. H., Dougherty, M. K., Lamy, L., Provan, G., and Zarka, P.: Planetary period oscillations in Saturn's magnetosphere: Evidence in magnetic field phase data for rotational modulation of Saturn kilometric radiation emissions, J. Geophys. Res., 116, A09206, doi:10.1029/2011JA016636, 2011.

Andrews, D. J., Cowley, S. W. H., Dougherty, M. K., Lamy, L., Provan, G., and Southwood, D. J.: Planetary period oscillations in Saturn's magnetosphere: Evolution of magnetic oscillation properties from southern summer to post-equinox, J. Geophys. Res., 117, A04224, doi:10.1029/2011JA017444, 2012.

Balachandran, N. K.: Gravity waves from thunderstorms, Mon. Weather Rev., 108, 804-816, doi:10.1175/15200493(1980)108<0804:GWFT>2.0.CO;2, 1980.

Carbary, J. F., Mitchell, D. G., Krimigis, S. M., and Krupp, N.: Dual periodicities in energetic electrons at Saturn, Geophys. Res. Lett., 36, L20103, doi:10.1029/2009GL040517, 2009.

Carbary, J. F., Mitchell, D. G., Krimigis, S. M., and Krupp, N.: Postequinox periodicities in Saturn's energetic electrons, Geophys. Res. Lett., 38, L24104, doi:10.1029/2011GL050259, 2011.

Cowley, S. W. H. and Provan, G.: Saturn's magnetospheric planetary period oscillations, neutral atmosphere circulation, and thunderstorm activity: Implications, or otherwise, for physical links, J. Geophys. Res., 118, 7246-7261, doi:10.1002/2013JA019200, 2013.

Davies, M. E., Abalakin, V. K., Bursa, M., Lieske, J. H., Morando, B., Morrison, D., Seidelmann, P. K., Sinclair, A. T., Yallop, B., and Tjuflin, Y. S.: Report of the IAU/COSPAR working group on cartographic coordinates and rotational elements of the planets and satellites: 1994, Cel. Mech. Dyn. Astron., 63, 127-148, doi:10.1007/BF00693410, 1996.

Desch, M. D. and Kaiser, M. L.: Voyager measurements of the rotation period of Saturn's magnetic field, Geophys. Res. Lett., 8, 253-256, doi:10.1029/GL008i003p00253, 1981.

Dyudina, U. A., Ingersoll, A. P., Ewald, S. P., Porco, C. C., Fischer, G., and Yair, Y.: Saturn's visible lightning, its radio emissions, and the structure of the 2009-2011 lightning storms, Icarus, 226, 1020-1037, doi:10.1016/j.icarus.2013.07.013, 2013.

Fischer, G., Gurnett, D. A., Kurth, W. S., Akalin, F., Zarka, P., Dyudina, U. A., Farrell, W. M., and Kaiser, M. L.: Atmospheric electricity at Saturn, Space Sci. Rev., 137, 271-285, doi:10.1007/s11214-008-9370-z, 2008.

Fischer, G., Kurth, W. S., Gurnett, D. A., Zarka, P., Dyudina, U. A., Ingersoll, A. P., Ewald, S. P., Porco, C. C., Wesley, A., Go, C., and Delcroix, M.: A giant thunderstorm on Saturn, Nature, 475, 75-77, doi:10.1038/nature10205, 2011.

Fischer, G., Gurnett, D. A., Kurth, W. S., Ye, S.-Y., and Groene, J. B.: Saturn kilometric radiation periodicity after equinox, Icarus, submitted, 2014.

Fletcher, L. N., Hesman, B. E., Irwin, P. G. J., Baines, K. H., Momary, T. W., Sánchez-Lavega, A., Flasar, M., Read, P., Orton, G. S., Simon-Miller, A., Hueso, R., Bjoraker, G. L., Mamoutkine, A., del Rio-Gaztelurrutia, T., Gomez, J. M., Buratti, B., Clark, R. N., Nicholson, P. D., and Sotin, C.: Thermal structure and dynamics of Saturn's northern springtime disturbance, Science, 332, 1413-1417, doi:10.1126/science.1204774, 2011.

Fletcher, L. N, Hesman, B. E., Achterberg, R. K., Irwin, P. G. J., Bjoraker, G., Gorius, N., Hurley, J., Sinclair, J., Orton, G. S., Legarreta, J., García-Melendo, E., Sánchez-Lavega, A., Read, P., Simon-Miller, A. A., and Flasar, M.: The origin and evolution of Saturn's 2011-2012 stratospheric vortex, Icarus, 221, 560-586, doi:10.1016/j.icarus.2012.08.024, 2012.

Friedson, A. J. and Moses, J. I.: General circulation and transport in Saturn's upper troposphere and stratosphere, Icarus, 218, 861875, doi:10.1016/j.icarus.2012.02.004, 2012.

Galopeau, P. and Lecacheux, A.: Variations of Saturn's radio rotation period measured at kilometer wavelengths, J. Geophys. Res., 105, 13089-13101, doi:10.1029/1999JA005089, 2000.

García-Melendo, E., Hueso, R., Sánchez-Lavega, A., Legarreta, J., del Río-Gaztelurrutia, T., Pérez-Hoyos, S., and Sanz-Requena, J. F.: Atmospheric dynamics of Saturn's 2010 giant storm, Nat. Geosci., 6, 525-529, doi:10.1038/ngeo1860, 2013.

Gurnett, D. A., Kurth, W. S., Kirchner, D. L., Hospodarsky, G. B., Averkamp, T. F., Zarka, P., Lecacheux, A., Manning, R., Roux, A., Canu, P., Cornilleau-Wehrlin, N., Galopeau, P., Meyer, A., Boström, R., Gustafsson, G., Wahlund, J.-E., Ahlen, L., Rucker, H. O., Ladreiter, H. P., Macher, W., Woolliscroft, L. J. C., Alleyne, H., Kaiser, M.L., Desch, M. D., Farrell, W. M., Harvey, C. C., Louarn, P., Kellogg, P. J., Goetz, K., and Pedersen, A.: The Cassini Radio and Plasma Wave investigation, Space Sci. Rev., 114, 395-463, doi:10.1007/s11214-004-1434-0, 2004.

Gurnett, D. A., Kurth, W. S., Hospodarsky, G. B., Persoon, A. M., Averkamp, T. F., Cecconi, B., Lecacheux, A., Zarka, P., Canu, P., Cornilleau-Wehrlin, N., Galopeau, P., Roux, A., Harvey, C., Louarn, P., Boström, R., Gustafsson, G., Wahlund, J.-E., Desch, M. D., Farrell, W. M., Kaiser, M. L., Goetz, K., Kellogg, P. J., Fischer, G., Ladreiter, H.-P., Rucker, H. O., Alleyne, H., and Pedersen, A.: Radio and plasma wave observations at Saturn from Cassini's approach and first orbit, Science, 307, 1255-1259, 2005.

Gurnett, D. A., Persoon, A. M., Kurth, W. S., Groene, J. B., Averkamp, T. F., Dougherty, M. K., and Southwood, D. J.: The variable rotation period of the inner region of Saturn's plasma disk, Science, 316, 442-445, doi:10.1126/science.1138562, 2007. 
Gurnett, D. A., Lecacheux, A., Kurth, W. S., Persoon, A. M., Groene, J. B., Lamy, L., Zarka, P., and Carbary, J. F.: Discovery of a north-south asymmetry in Saturn's radio rotation period, Geophys. Res. Lett., 36, L16102, doi:10.1029/2009GL039621, 2009.

Gurnett, D. A., Groene, J. B., Persoon, A. M., Menietti, J. D., Ye, S.-Y., Kurth, W. S., MacDowall, R. J., and Lecacheux, A.: The reversal of the rotational modulation rates of the north and south components of Saturn kilometric radiation near equinox. Geophys. Res. Lett., 37, L24101, doi:10.1029/2010GL045796, 2010.

Gurnett, D. A., Groene, J. B., Averkamp, T. F., Kurth, W. S., Ye, S.Y., and Fischer, G.: An SLS4 longitude system based on a tracking filter analysis of the rotational modulation of Saturn kilometric radiation, in: Planetary Radio Emissions VII, edited by: Rucker, H. O., Kurth, W. S., Louarn, P., and Fischer, G., Austrian Academy of Sciences Press, Vienna, 51-64, 2011.

Heimpel, M. and Aurnou, J. M.: Convective bursts and the coupling of Saturn's equatorial storms and interior rotation, Astrophys. J., 746, 51-65, doi:10.1088/0004-637X/746/1/51, 2012.

Helled, R., Schubert, G., and Anderson, J. D.: Empirical models of pressure and density in Saturn's interior: Implications for the helium concentration, its depth dependence, and Saturn's precession rate, Icarus, 199, 368-377, doi:10.1016/j.icarus.2008.10.005, 2009.

Hines, C. O.: Momentum deposition by atmospheric waves, and its effects on thermospheric circulation, The Upper Atmosphere in Motion, Geophys. Monograph Series, 18, American Geophys. Union, Washington DC, 760-768, 1974.

Huang, T. S. and Hill, T. W.: Corotation lag of the Jovian atmosphere, ionosphere, and magnetosphere, J. Geophys. Res., 94, 3761-3765, doi:10.1029/JA094iA04p03761, 1989.

Hurley, J., Irwin, P. G. J., Fletcher, L. N., Moses, J. I., Hesman, B., Sinclair, J., and Merlet, C.: Observations of upper tropospheric acetylene on Saturn: No apparent correlation with $2000 \mathrm{~km}$-sized thunderstorms, Planet. Space Sci., 65, 21-37, doi:10.1016/j.pss.2011.12.026, 2012.

Jia, X. and Kivelson, M. G.: Driving Saturn's magnetospheric periodicities from the upper atmosphere/ionosphere: Magnetotail response to dual sources, J. Geophys. Res., 117, A11219, doi:10.1029/2012JA018183, 2012.

Jia, X., Kivelson, M. G., and Gombosi, T. I.: Driving Saturn's magnetospheric periodicities from the upper atmosphere/ionosphere, J. Geophys. Res., 117, A04215, doi:10.1029/2011JA017367, 2012.

Kaiser, M. L., Desch, M. D., Kurth, W. S., Lecacheux, A., Genova, F., and Pedersen, B. M.: Saturn as a radio source, in: Saturn, edited by: Gehrels, T. and Matthews, M. S., Univ. Arizona Press, Tuscon, 378-415, 1984.

Kimura, T., Lamy, L., Tao, C., Badman, S., Kasahara, S., Cecconi, B., Zarka, P., Morioka, A., Miyoshi, Y., Maruno, D., Kasaba, Y., and Fujimoto, M.: Long-term modulations of Saturn's auroral radio emissions by the solar wind and seasonal variations controlled by the solar ultraviolet flux. J. Geophys. Res., 118, 70197035, doi:10.1002/2013JA018833, 2013.

Kurth, W. S., Lecacheux, A., Averkamp, T. F., Groene, J. B., and Gurnett, D. A.: A Saturnian longitude system based on a variable kilometric radiation period, Geophys. Res. Lett., 34, L02201, doi:10.1029/2006GL028336, 2007.
Kurth, W. S., Averkamp, T. F., Gurnett, D. A., Groene, J. B., and Lecacheux, A.: An update to a Saturnian longitude system based on kilometric radio emissions, J. Geophys. Res., 113, A05222, doi:10.1029/2007JA012861, 2008.

Lamy, L.: Variability of southern and northern SKR periodicities, in: Planetary Radio Emissions VII, edited by: Rucker, H. O., Kurth, W. S., Louarn, P., and Fischer, G., Austrian Academy of Sciences Press, Vienna, 39-50, 2011.

Lamy, L., Zarka, P., Cecconi, B., Prangé, R., Kurth, W. S., and Gurnett, D. A.: Saturn kilometric radiation: Average statistical properties, J. Geophys. Res., 113, A07201, doi:10.1029/2007JA012900, 2008a.

Lamy, L., Zarka, P., Cecconi, B., Hess, S., and Prangé, R.: Modeling of Saturn kilometric radiation arcs and equatorial shadow zone, J. Geophys. Res., 113, A10213, doi:10.1029/2008JA013464, 2008b.

Matcheva, K. I. and Barrow, D. J.: Small-scale variability in Saturn's lower ionosphere, Icarus, 221, 525-543, doi:10.1016/j.icarus.2012.08.022, 2012.

Miyoshi, Y. and Fujiwara, H.: Gravity waves in the thermosphere simulated by a General Circulation Model, J. Geophys. Res., 113, D01101, doi:10.1029/2007JD008874, 2008.

Müller-Wodarg, I. C. F., Mendillo, M., Yelle, R. V., and Aylward, A. D.: A global circulation model of Saturn's thermosphere, Icarus, 180, 147-160, doi:10.1016/j.icarus.2005.09.002, 2006.

Pierce, A. D. and Coroniti, S. C.: A mechanism for the generation of acoustic-gravity waves during thunderstorm formation, Nature, 210, 1209-1210, doi:10.1038/2101209a0, 1966.

Provan, G., Cowley, S. W. H., Sandhu, J., Andrews, D. J., and Dougherty, M. K.: Planetary period magnetic field oscillations in Saturn's magnetosphere: Postequinox abrupt nonmonotonic transitions to northern system dominance, J. Geophys. Res., 118, 3243-3264, doi:10.1002/jgra.50186, 2013.

Sánchez-Lavega, A.: Saturn's Great White Spots, Chaos, 4, 341353, doi:10.1063/1.166012, 1994.

Sánchez-Lavega, A., del Rio-Gaztelurrutia, T., Hueso, R., GmezForrellad, J. M., Sanz-Requena, J. F., Legarreta, J., GarcíaMelendo, E., Colas, F., Lecacheux, J., Fletcher, L. N., Barrado y Navascués, D., Parker, D., and the international outer planet watch team: Deep winds beneath Saturn's upper clouds from a seasonal long-lived planetary-scale storm, Nature, 475, 71-74, doi:10.1038/nature10203, 2011.

Sánchez-Lavega, A., del Rio-Gaztelurrutia, T., Delcroix, M., Legarreta, J., Gmez-Forrellad, J. M., Hueso, R., García-Melendo, E., Pérez-Hoyos, S., Barrado-Navascués, D., Lillo, J., and the international outer planet watch team IOPW-PVOL: Groundbased observations of the long-term evolution and death of Saturn's 2010 Great White Spot, Icarus, 220, 561-576, doi:10.1016/j.icarus.2012.05.033, 2012.

Sayanagi, K. M. and Showman, A. P.: Effects of a large convective storm on Saturn's equatorial jet, Icarus, 187, 520-539, doi:10.1016/j.icarus.2006.10.020, 2007.

Sayanagi, K. M., Dyudina, U. A., Ewald, S. P., Fischer, G., Ingersoll, A. P., Kurth, W. S., Muro, G. D., Porco, C. C., and West, R. A.: Dynamics of Saturn's great storm of 20102011 from Cassini ISS and RPWS, Icarus, 223, 460-478, doi:10.1016/j.icarus.2012.12.013, 2013.

Schubert, G., Hickey, M. P., and Walterscheid, R. L.: Heating of Jupiter's thermosphere by the dissipation of upward propagat- 
ing acoustic waves, Icarus, 163, 398-413, doi:10.1016/S00191035(03)00078-2, 2003.

Smith, C. G. A.: Periodic modulation of gas giant magnetospheres by the neutral upper atmosphere, Ann. Geophys., 24, 2709-2717, doi:10.5194/angeo-24-2709-2006, 2006.

Smith, C. G. A.: A Saturnian cam current system driven by asymmetric thermospheric heating, Mon. Not. R. Astron. Soc., 410, 2315-2328, doi:10.1111/j.1365-2966.2010.17602.x, 2011.

Southwood, D. J. and Kivelson, M. G.: The source of Saturn's periodic radio emission, J. Geophys. Res., 114, A09201, doi:10.1029/2008JA013800, 2009.

Stull, R. B.: Internal gravity waves generated by penetrative convection, J. Atmos. Sci., 33, 1279-1286, doi:10.1175/15200469(1976)033<1279:IGWGBP>2.0.CO;2, 1976.

Vadas, S. L. and Liu, H.: Generation of large-scale gravity waves and neutral winds in the thermosphere from the dissipation of convectively generated gravity waves, J. Geophys. Res., 114, A10310, doi:10.1029/2009JA014108, 2009.
Ye, S.-Y., Menietti, J. D., Fischer, G., Wang, Z., Cecconi, B., Gurnett, D. A., and Kurth, W. S.: Z mode waves as the source of Saturn narrowband radio emissions, J. Geophys. Res., 115, A08228, doi:10.1029/2009JA015167, 2010a.

Ye, S.-Y., Gurnett, D. A., Groene, J. B., Wang, Z., and Kurth, W. S.: Dual periodicities in the rotational modulation of Saturn narrowband emissions, J. Geophys. Res., 115, A12258, doi:10.1029/2010JA015780, 2010b.

Yelle, R. V. and Miller, S.: Jupiter's thermosphere and ionosphere, in: Jupiter. The planet, satellites and magnetosphere, edited by: Bagenal, F., Dowling, T. E., and McKinnon, W. B., Cambridge Univ. Press, Cambridge, UK, 185-218, 2004.

Yiğit, E., Medvedev, A. S., Aylward, A. D., Ridley, A. J., Harris, M. J., Moldwin, M. B., and Hartogh, P.: Dynamical effects of internal gravity waves in the equinoctial thermosphere, J. Atmos. Sol.-Terr. Phys., 90-91, 104-116, doi:10.1016/j.jastp.2011.11.014, 2012.

Zarka, P., Lamy, L., Cecconi, B., Prangé, R., and Rucker, H. O.: Modulation of Saturn's radio clock by solar wind speed, Nature, 450, 265-267, doi:10.1038/nature06237, 2007. 